\title{
Risque suicidaire et stress chez les jeunes homosexuels
}

\section{Stress and suicidal risk among homosexuals}

\author{
Charbonnier Elodie, Coralie Hatteschweiler et Pierluigi Graziani
}

\section{Résumé :}

L'objectif principale de cette recherche est d'appréhender la manière dont les jeunes homosexuels évaluent et font face à une situation hautement stressante. Notre second objectif est de préciser les liens entre stress et risque suicidaire chez les jeunes homosexuels. Un questionnaire s'inspirant du «Questionnaire d'Évaluation de la Transaction Stressante» a été administré à 200 jeunes homosexuels. Les participants ont répondu à cet auto-questionnaire en s'appuyant sur la situation la plus stressante de leur vie. Parmi l'ensemble de nos participants, 52 ont choisi l'annonce de leur homosexualité. Ils ont été regroupés dans le groupe « $\mathrm{CO} »$ (coming-out). Les 148 autres sujets ont été inclus dans le groupe « AS » (autre situation). Cette recherche met en évidence le risque suicidaire élevé des jeunes homosexuels lorsqu'ils s'exposent à une situation très stressante. Des prises en charge s'inspirant des programmes de gestion du stress pourraient donc être des axes de prévention du suicide intéressants à développer auprès de ces jeunes qui ont un risque suicidaire plus élevé que les hétérosexuels.

Mots clés : homosexuels, stress, risque suicidaire, annonce de son homosexualité

\begin{abstract}
Abtract :
Stress is omnipresent in the homosexual community. This research aimed to understand how young homosexuals can assess and cope with highly stressful situations. The stress generated by the revelation of homosexuality has been especially studied since, for many homosexuals, the experience of coming-out is highly emotional. A questionnaire adapted from the "Questionnaire d'Évaluation de la Transaction Stressante (QETS)» and based on the transactional model of stress has been filled by 200 young homosexuals. We assessed the characteristics of stressful situations, the risks that participants considered present in those situations (primary endpoint), and the action possibilities which they could use to confront them (secondary endpoint). We evaluated the presence of suicidal ideas, scenarios and behaviors during stressful situations, and also anxiety and depression symptoms that were felt during the questionnaire session. Young homosexuals then reported the most stressful situation they have encountered during their lifetime. Announcing their homosexuality was chosen by 52 of the total sample. From this observation, two groups were formed. The first one consisted of the 52 young homosexuals who considered their coming out to be the most stressful situation they have ever encountered («CO group »; coming out). The second one consisted of the other 148 participants («AS group »; other situation). Young homosexuals in the « CO group » were significantly more fearful that this situation could hurt their families. Furthermore, they felt more passive than the participants in the "AS group ». Our results also showed the prevalence of suicidal ideas, scenarios and behaviors amongst young homosexuals after facing very stressful situations. These results open a new line of research in terms of homosexual's suicide prevention. They underline the necessity to develop programs based on stress management in order to prevent suicidal behaviors amongst homosexuals.
\end{abstract}

Keywords: homosexuals, stress, suicidal risk, coming-out 


\section{Introduction}

Cette recherche quantitative a été menée auprès de 200 jeunes se disant homosexuels. Le premier objectif de cette recherche est d'appréhender la manière dont les jeunes homosexuels évaluent un événement hautement stressant, et notamment la divulgation de leur homosexualité. Notre second objectif vise à déterminer les liens existants entre le stress et le risque suicidaire chez les jeunes homosexuels. Cette recherche s'appuie essentiellement sur deux conceptions théoriques : le «modèle des stresseurs des groupes minoritaires » (Meyer, 2003; Meyer, Dietrich, \& Schwartz, 2008; Meyer \& Northridge, 2007) et le «modèle transactionnel du stress » (Coyne \& Lazarus, 1980; Lazarus, 1966; Lazarus \& Folkman, 1984).

De nombreuses recherches mettent en évidence les différences entre les homosexuels et les hétérosexuels, notamment en ce qui concerne leur passage à l'acte suicidaires (Remafedi, 2002), leurs idéations suicidaires (Silenzio, Pena, Duberstein, Cerel, \& Knox, 2007) ou encore leur taux de dépression (Saewyc et al., 2007). La prévalence de leurs idées suicidaires et de leur tentative de suicide se retrouve dans de multiples recherches menées dans différents pays (Saewyc, 2011). En France, les données sont moins nombreuses mais elles confirment les études réalisées notamment aux Etats Unis et au Canada. Une des premières enquêtes françaises (Adam, 2001) a montré que 17\% des gays ont déjà fait une tentative de suicide au cours de leur vie, ce taux étant d'autant plus marqué chez les plus jeunes (27\% pour les moins de 20 ans). Cette enquête a été reconduite quelques années plus tard, et la part des hommes qui ont réalisé une tentative de suicide a augmenté entre 2000 et 2004, atteignant un taux de 19\% pour la totalité de l'échantillon et de 32\% pour les moins de 20 ans (Velter, 2007). Dans le cadre du « Baromètre Santé 2005 », une enquête française a été menée auprès de 16883 
sujets (15-75 ans) et rend compte de l'importance des tentatives de suicide chez les gays et bisexuels en comparaison aux hétérosexuels (7,5\% vs 2,5\%) (Jouvin, Beaulieu-Prévost, \& Julien, 2007). La recherche menée par Lhomond et Saurel-Cubizolles (2003) montre quant à elle que $25 \%$ des femmes (25-59 ans) qui ont eu des rapports avec d'autres femmes ont fait une tentative de suicide au cours de leur vie alors que ce taux est de $6 \%$ pour les femmes hétérosexuelles.

Leur risque suicidaire élevé peut être mis en lien avec le fait que les homosexuels possèdent moins de facteurs de protection que les hétérosexuels (Saewyc et al., 2009). Ils présentent également plus de facteurs de risque, comme par exemple un manque de soutien, des difficultés scolaires, des problèmes familiaux (Kitts, 2005), mais aussi une peur du harcèlement, des mauvais traitements (Huebner, Rebchook, \& Kegeles, 2004), de la stigmatisation et de la discrimination (Garofalo \& Katz, 2001). Une grande partie de la littérature se centre donc sur les facteurs environnementaux, les expériences de vie liées à leur appartenance à une minorité, pour expliquer le risque suicidaire élevé des jeunes homosexuels. Pour Hammelman (1993), il serait lié aux expériences de vie stressantes qu'ils rencontrent, que ce soit les expériences homophobes ou les réactions négatives qui font suite à la divulgation de leur homosexualité, d'autant plus lorsqu'elles proviennent de personnes importantes pour eux.

Selon le «modèle des stresseurs des groupes minoritaires » (Meyer, 2003), le risque suicidaire élevé des homosexuels peut être mis en lien avec l'excès de stress qu'ils ressentent. Ces auteurs défendent l'idée que les personnes appartenant à un groupe minoritaire présenteraient un stress marqué (Meyer \& Northridge, 2007). Celui-ci serait aux stresseurs spécifiques liés à leur appartenance à un groupe minoritaire qui viendraient s'ajouter aux 
stresseurs généraux rencontrés par la population générale. Parmi les stresseurs spécifiques des homosexuels, nous retrouvons par exemple le fait d'annoncer son orientation sexuelle, qui est une situation à laquelle seuls les homosexuels sont confrontés et qui peut s'avérer hautement stressante (D'Augelli, 2002; Floyd \& Stein, 2002). Selon ce modèle, l'accumulation des stresseurs permettrait en partie de comprendre le risque suicidaire élevé des jeunes homosexuels.

Nous avons fait le choix de nous centrer sur la divulgation de leur homosexualité car cette situation présente des enjeux identitaires, sociétaux et familiaux. Elle implique de se définir comme différent, c'est-à-dire comme gay, lesbienne ou bisexuel (Bonet, Wells, \& Parsons, 2007), mais également de se confronter au regard de l'autre suite à cette divulgation et à son éventuel jugement. En effet, le stress lié à cette annonce est accentué par la possibilité qu'elle conduise à un rejet (D'Augelli, 2002; Meyer, 2003). De nombreux homosexuels n'annoncent pas leur homosexualité, notamment à leurs parents, par peur que cette révélation ne modifie leurs relations avec eux (D'Augelli, Grossman, Starks, \& Sinclair, 2010). Leurs inquiétudes sont d'autant plus nombreuses lorsqu'ils vivent avec leurs parents, qu'ils dépendent d'eux financièrement, ou qu'ils appartiennent à une autre minorité (Savin-Williams \& Esterberg, 2000). Suite à cette révélation, certains jeunes sont parfois exclus de leur domicile, d'autres préfèrent s'en aller au vu du contexte familial conflictuel (Beck, Firdion, Legleye, \& Schiltz, 2010). Mais au-delà des conséquences matérielles, les conséquences psychologiques d'un rejet sont aussi importantes. Lorsque cette divulgation conduit à un rejet parental, elle peut alors favoriser un développement caractérisé par l'instabilité, la peur et l'anxiété (Julien, 2000). Dans l'étude réalisée par Ryan et ses collaborateurs (2009), 43 \% des sujets dont les parents ont eu des réactions négatives déclarent avoir eu des idées suicidaires, le taux est de $11 \%$ chez les jeunes dont les parents ont bien accepté leur homosexualité. 
Dévoiler son homosexualité peut donc constituer un moment difficile et stressant (SavinWilliams, 2001; Willoughby \& Malik, 2006a). Cependant, cette annonce n'est pas forcément un stresseur négatif (Iwasaki \& Ristock, 2007). Lorsqu'elle est bien accueillie par l'entourage, elle représente un critère d'accession à une identité sexuelle positive (Julien, 2000) et favorise le bien-être (Floyd, Stein, Harter, Allison, \& Nye, 1999). De plus, les réactions positives sont corrélées négativement avec le risque de réaliser une tentative de suicide (Eisenberg \& Resnick, 2006).

Ces données illustrent la complexité de cette annonce. Afin de mieux comprendre les caractéristiques du stress des jeunes homosexuels lors de la divulgation de leur homosexualité, nous nous sommes également appuyés sur le «modèle transactionnel du stress » développé par Lazarus et ses collaborateurs. Le «modèle transactionnel du stress » (Coyne \& Lazarus, 1980; Lazarus, 1966; Lazarus \& Folkman, 1984) met l'accent sur la façon dont le sujet construit la transaction stressante. Dans cette approche, ce n'est pas l'événement qui est considéré comme générateur de stress mais l'évaluation cognitive faite par le sujet. Le stress dépend donc de la manière dont le sujet interprète la situation (stress perçu) et analyse sa capacité à y faire face (contrôle perçu). Par conséquent, le stress est présent lorsque le sujet perçoit la situation comme dangereuse et qu'il ne se sent pas capable d'y faire face.

\section{Méthodologie}

Afin d'appréhender la manière dont les jeunes homosexuels évaluent un événement hautement stressant, un questionnaire s'inspirant du «Questionnaire d'Évaluation de la Transaction Stressante » a été administré à 200 jeunes se disant homosexuels. Pour identifier les liens entre le stress et le risque suicidaire au sein de cette population, nous l'avons 
complété par des échelles évaluant l'anxiété, la dépression et le risque suicidaire. Cette étude a été réalisée dans le cadre d'un doctorat, elle a fait l'objet d'une supervision de la part d'un Professeur Universitaire en psychologie clinique et psychopathologie.

\subsection{Population d'étude}

\subsubsection{Ensemble de l'échantillon}

Les personnes interrogées ont toutes été recrutées sur internet, via différents sites de rencontre spécialisés pour les personnes lesbiennes, gays ou bisexuelles (Gaypax ${ }^{\circledR}$, Gayvox ${ }^{\circledR}$ ). Des messages privés ont été envoyés à l'ensemble des profils en précisant la thématique de l'étude, le cadre de la recherche, ainsi que la procédure à suivre. Les personnes intéressées nous ont envoyé un mail et un questionnaire confidentiel leur a été retourné, après qu'ils aient signé un formulaire de consentement. Une fois le questionnaire rempli et retourné, ils ont été recontactés (par mail ou téléphone), afin de vérifier le déroulement de leur passation, de leur expliquer la recherche et de répondre à leurs éventuelles questions. Le recrutement par internet constitue aujourd'hui un mode de recrutement efficace lorsqu'on s'intéresse à une population spécifique (Fenner et al., 2012) (comme c'est le cas des personnes homosexuelles) ce qui a amené de nombreux organismes de recherche français (INSERM et IVS) à l'utiliser (Pouchieu, Castetbon, Galan, Hercberg, \& Touvier, 2012). Ce mode de recrutement nous est apparu pertinent afin de constituer un échantillon conséquent et varié. Se limiter aux homosexuels fréquentant les associations ou lieux homosexuels nous semblait être une méthode de recrutement ne permettant pas d'accéder à un échantillon diversifié.

$\mathrm{Au}$ début du questionnaire, il a été demandé aux sujets de définir leur orientation sexuelle. Nous avons conservé les questionnaires dans lesquels les participants ont répondu 
être « homosexuel-le» (174 sujets) ou « bisexuel-le» (26 sujets). En l'absence de différence significative entre les résultats des homosexuels et des bisexuels, et pour simplifier la lisibilité du texte, le terme «homosexuel» sera utilisé dans l'ensemble de l'article pour tous les participants. Le recrutement a été arrêté une fois notre objectif atteint de 200 questionnaires valides. Les participants ont entre 18 et 26 ans. Cette tranche d'âge a été choisie car c'est principalement au cours de cette période que les homosexuels commencent à annoncer leur homosexualité à leurs proches (Savin-Williams \& Ream, 2003b). La moyenne d'âge de l'ensemble de l'échantillon est de 21,90 ans $(\mathrm{ET}=2,65)$. L'échantillon total se compose de 76 lesbiennes (38\% de l'échantillon total) et de 124 gays (62\% de l'échantillon total). Il y a significativement plus d'hommes que de femmes $\left(\chi_{1}^{2}=11,52 ; \mathrm{p}=0,001\right)$. Les participants déclarent avoir déjà annoncé leur homosexualité en moyenne 4 fois $(\mathrm{ET}=1,56)$ et avoir vécu en moyenne 2,42 annonces stressantes $(\mathrm{ET}=1,75)$.

\subsubsection{Répartition des sujets}

La consigne proposée à l'ensemble des participants au début du questionnaire était la suivante : "souvenez-vous de l'événement le plus stressant que vous avez rencontré dans votre vie et répondez aux questions suivantes ». Ce questionnaire a été administré à 200 jeunes. Parmi eux, 52 sujets (soit 26\%) ont cité l'annonce de leur homosexualité comme étant la situation la plus stressante de leur vie et se sont basés sur cette divulgation pour répondre au questionnaire. Afin d'identifier les spécificités du stress lors de la révélation de leur homosexualité, ces sujets ont été mis dans le groupe intitulé « $\mathrm{CO} »($ Coming-Out). En moyenne, les jeunes de ce groupe ont affirmé avoir annoncé 4,31 fois leur orientation sexuelle au cours de leur vie $(\mathrm{ET}=1,63)$ et avoir rencontré en moyenne 2,96 annonces stressantes $(\mathrm{ET}$ $=1,79)$. 
Les autres participants ont été inclus dans le groupe «AS» (Autre Situation) qui est donc composé des 148 autres sujets restants qui ont évoqué d'autres situations stressantes. Les situations citées dans ce groupe sont très hétérogènes et ne sont pas toutes liées à leur orientation sexuelle. Les problèmes au sein de la famille sont les situations les plus fréquemment évoquées (36 jeunes soit 24,32\%), vient ensuite les problèmes sentimentaux pour 18,24\% des sujets (27 jeunes) et les problèmes de santé (17 jeunes soit 11,49\%). Des problèmes au travail ont été cités par 9 jeunes $(6,10 \%), 7$ ont évoqué leurs études, 7 ont parlé de leurs loisirs, 7 autres ont cité des évènements en lien avec les transports. Des situations très spécifiques ont été citées par seulement quelques sujets, ce qui ne nous a pas permis de les regrouper (par exemple des problèmes légaux ont été évoqués par 4 d'entre eux). Par conséquent, au sein de notre échantillon, les stresseurs généraux sont évoqués plus fréquemment que les stresseurs spécifiques. Il est cependant important de noter que près d'un quart ont évoqués la même situation : l'annonce de leur homosexualité.

Même si les jeunes du groupe «AS»n'ont pas évoqué la divulgation de leur homosexualité comme étant la situation la plus stressante de leur vie, c'est malgré tout une situation qu'ils ont presque tous rencontré (seulement 5 jeunes n'ont jamais divulgué leur homosexualité). Ils ont déclaré en moyenne avoir révélé 3,93 fois leur homosexualité au cours de leur vie $(E T=1,63)$, les sujets des deux groupes ont donc annoncé autant de fois leur homosexualité $\left(\mathrm{t}{ }_{(198)}=1,53 ; \mathrm{p}=\mathrm{NS}\right)$. En moyenne dans le groupe « AS », les jeunes ont affirmé avoir vécu 2,23 annonces stressantes $(\mathrm{ET}=1,69)$, ils ont donc rencontré significativement moins d'annonces stressantes que ceux du groupe « $\mathrm{CO} »\left(\mathrm{t}_{(198)}=2,62 ; \mathrm{p}=\right.$ 0,009). 
Nous n'observons pas de différence d'âge statistiquement significative entre les deux groupes $\left(\mathrm{m}_{\mathrm{AS}}=21,88 ; \mathrm{m}_{\mathrm{CO}}=21,96 ; \mathrm{t}_{(198)}=0,19 ; \mathrm{p}=\mathrm{NS}\right)$. Dans le groupe $« \mathrm{AS} »$, la répartition entre les sexes est statistiquement identique $\left(\chi^{2}{ }_{1}=3,27 ; \mathrm{p}=\mathrm{NS}\right)$. Par contre, le groupe « $\mathrm{CO} »$ est composé de significativement plus d'hommes (39 gays soit 75\% du groupe «CO ») que de femmes (13 lesbiennes soit $25 \%$ du groupe « $\mathrm{CO} »)\left(\mathcal{X}_{1}^{2}=13 ; \mathrm{p}=0,0001\right)$. Par conséquent, l'annonce de son homosexualité est plus fréquemment une situation hautement stressante chez les gays que chez les lesbiennes. En effet, dans notre échantillon total, $31,5 \%$ des hommes considèrent que la divulgation de leur homosexualité a été la situation la plus stressante de leur vie, alors que ce n'est le cas que de 17,1 \% des femmes.

\subsection{Variables étudiées}

Afin d'identifier la manière dont les jeunes homosexuels évaluent la situation la plus stressante de leur vie, un questionnaire s'inspirant du «Questionnaire d'Évaluation de la Transaction Stressante (QETS)» (Graziani, Rusinek, Servant, Hautekèete-Sence, \& Hautekèete, 1998) leur a été proposé. Il a été demandé aux participants de se remémorer l'événement le plus stressant qu'ils ont rencontré dans leur vie et d'évaluer les risques qu'ils pensaient encourir (évaluation primaire), les moyens d'action dont ils pensaient bénéficier (évaluation secondaire) et les caractéristiques de la situation. Leurs symptômes anxiodépressifs au moment de la passation et leur risque suicidaire ont également été évalués. 


\subsubsection{Caractéristiques de la situation stressante}

Les sujets ont précisé leur âge au moment de la transaction stressante, le niveau de répétition de cette situation dans le passé (sur une échelle allant de « jamais » à « cinq fois ou plus »), ainsi que leur degré de préparation et d'initiative (sur une échelle allant de 0 « pas du tout» à 4 «tout à fait»). Ils ont également évalué quel a été le résultat de cette situation. Plusieurs modalités de réponse leur ont été proposées allant de "pas de solution et la situation s'est empirée » à "résolue comme vous l'avez desiré ». Nous les avons également questionnés sur les personnes qui étaient impliquées dans la situation stressante. Ils avaient plusieurs choix de réponses possibles (leurs parents, leurs amis, leurs collègues de travail, leurs supérieurs, d'autres membres de leur famille,...).

\subsubsection{Evaluation des risques encourus}

Selon le «modèle transactionnel du stress », lorsque nous nous confrontons à une situation, nous évaluons dans un premier temps la menace éventuelle encourue (évaluation primaire). Afin de préciser cette menace, les participants ont évalué l'intensité de 13 risques généraux, sur une échelle allant de 0 pour « pas du tout » à 4 pour « beaucoup » :

- Nuire au bien-être physique, et à la santé d'un de vos proches

- Faire de la peine à un proche

- Nuire à votre propre santé physique, à votre sécurité ou à votre bien-être

- Perturber les habitudes d'un proche

- Ne pas réussir un but important au travail

- Diminuer vos ressources financières

- Perdre l'affection de quelqu'un d'important pour vous 
- Perdre votre amour propre

- Apparaître comme quelqu'un d'insouciant

- Apparaître comme quelqu'un sans moralité

- Perdre l'approbation ou le respect de quelqu'un d'important pour vous

- Perdre le respect pour quelqu'un d'autre

- Vous montrer incompétent

\subsubsection{Evaluation des possibilités d'action}

Suite à cette première évaluation, ils ont estimé les moyens d'action qu'ils pensaient avoir sur la situation (évaluation secondaire). Cette seconde évaluation a été opérationnalisée en quatre possibilités d'intervention: " changer la situation ou y faire quelque chose », « l'accepter», « en savoir plus avant d'agir», ou «se retenir de faire ce qu'ils auraient voulu ». Ces quatre possibilités d'action ont été évaluées sur une échelle en 5 points allant de 0 « pas du tout » à 4 « beaucoup ».

\subsubsection{Idéations et tentatives de suicide}

Il a également été demandé aux participants de préciser la présence d'idées, de scénarios ou encore de passages à l'acte suicidaires, au cours de la situation la plus stressante de leur vie. Pour chaque question, les réponses ont été regroupées en deux catégories : « Oui » et « Non ». Les symptômes anxieux et dépressifs au moment de la passation ont également été étudiés. Pour cela, les sujets ont répondu à l' "Hospital Anxiety and Depression scale » (HADS) (Zigmond \& Snaith, 1983). Cette échelle d'auto-évaluation est composée de 14 items, elle évalue la symptomatologie anxieuse et dépressive. La passation de la HADS a été 
associée à la «Suicide Behaviors Questionnaire-Revised»(SBQ-R). Cette seconde échelle a été traduite en français pour cette recherche, suite à l'accord de l'auteur. Elle est composée de 4 items portant sur les idées et les comportements suicidaires et évalue le risque suicidaire du sujet. Elle a été testée et validée sur des sujets présentant une pathologie psychiatrique (adolescents et adultes), ainsi que sur la population générale (adolescents et adultes) (Osman et al., 2001). Elle permet de discriminer les individus présentant un risque suicidaire et ceux qui n'en présentent pas.

\section{Résultats}

\subsection{Caractéristiques de la situation stressante}

\section{- Répétition, préparation et initiative}

En moyenne, les jeunes interrogés ont été confrontés à la situation la plus stressante de leur vie à 18,73 ans $(\mathrm{ET}=3,38)$, il y a en moyenne 3,17 ans $(\mathrm{ET}=3,12)$. Il n'y a pas de différence entre les deux groupes en ce qui concerne l'âge qu'ils avaient au moment de la situation $\left(\mathrm{t}_{(198)}=0,51 ; \mathrm{p}=\mathrm{NS}\right)$. Ceux qui considèrent que l'annonce de leur homosexualité a été la situation la plus stressante de leur vie (groupe « $\mathrm{CO} »)$ ont plus fréquemment prévu et provoqué la situation, en comparaison de ceux qui ont répondu au questionnaire à partir d'une autre situation stressante (groupe « AS ») (cf tableau I). En effet, dans le groupe « $\mathrm{CO} »$, $34,61 \%$ déclarent l'avoir « tout à fait » prévu contre seulement $12,16 \%$ dans le groupe « AS ». De plus, $42,30 \%$ des sujets du groupe « $\mathrm{CO}$ » ont «tout à fait » été à l'initiative de la situation, alors que ce n'est le cas que de $18,92 \%$ des jeunes du groupe « AS ». L'annonce de son homosexualité apparait donc être une situation qui fait l'objet d'une plus grande préparation et qui est provoquée par le jeune, ce qui différencie cette situation des situations stressantes du quotidien choisies par les sujets du groupe « AS ». 
Tableau I : caractéristiques de la situation stressante, en fonction des deux groupes de sujets (moyennes, écarts-types et $t$ de student).

\begin{tabular}{|l|c|c|c|c|}
\hline & $\begin{array}{c}\text { Groupe « AS } \\
\mathbf{N}=\mathbf{1 4 8}\end{array}$ & $\begin{array}{c}\text { Groupe « CO } \\
\mathbf{N}=\mathbf{5 2}\end{array}$ & $\mathbf{t}_{(\mathbf{1 9 8})}$ & $\mathbf{p}$ \\
\hline Age & $18,80(3,65)$ & $18,52(2,47)$ & 0,51 & NS \\
\hline Répétition & $0,49(1,13)$ & $0,37(0,77)$ & 0,71 & NS \\
\hline Prévisibilité & $1,23(1,44)$ & $2,33(1,53)$ & 4,65 & 0.0001 \\
\hline Initiative & $1,30(1,61)$ & $2,44(1,61)$ & 4,38 & 0.0001 \\
\hline
\end{tabular}

\section{- Personnes impliquées}

Les participants ont précisé les personnes qui étaient impliquées dans la situation la plus stressante de leur vie. Dans le groupe «AS », la disparité des réponses est importante. Pour 20,9\% des sujets de ce groupe, cette situation n'impliquait que leurs parents (31 sujets) et pour $10,8 \%$, elle concernait leur famille élargie (6 jeunes). En dehors du cercle familial, $12,8 \%$ des participants ont évoqué leurs amis (19 jeunes), d'autres leur partenaire (15 sujets soit $10,1 \%$ ). Le travail est le secteur le moins fréquemment cité ( 8 jeunes ont évoqué leur supérieur soit 5,4\%). Pour les autres participants du groupe « $\mathrm{AS} »$, des configurations très hétérogènes ont été évoquées, telles que partenaires/parents, parents/enseignant. Dans le groupe « $\mathrm{CO} »$, la répartition est plus homogène. La moitié des participants évoquent l'annonce faite à leurs parents. Pour 15,4\%, c'est l'annonce réalisée auprès de leur famille élargie qui s'est avérée être la plus stressante et $11,5 \%$ ont cité la divulgation à leurs parents et à leurs amis. Chez 23\% des sujets c'est la divulgation faite exclusivement leurs amis qui a été pour eux la plus stressante. Pour les deux groupes, se sont donc des personnes proches qui sont concernées, plus généralement des membres de la famille, plus précisément leurs parents. 


\section{- Issue de la situation}

Les participants ont également évalué l'issue de la situation. Dans le groupe « $\mathrm{CO} »$, aucun sujet n'a considéré que la situation s'était aggravée depuis l'annonce de leur homosexualité la plus stressante de leur vie (cf tableau II). De plus, 63,46\% des jeunes du groupe « $\mathrm{CO}$ » ont même jugé qu'elle s'était résolue favorablement. Dans le groupe « AS », la proportion de sujets considérant que la situation s'était dégradée est nettement plus élevée mais elle reste relativement faible $(10,1 \%)$. Tout comme dans le groupe « $\mathrm{CO}$ », en majorité, ils évaluent positivement l'issue de la situation.

Tableau II: Evaluation de l'issue de la situation stressante en fonction des deux groupes de sujets (effectifs et pourcentages).

\begin{tabular}{|l|c|c|}
\hline \multicolumn{1}{|c|}{ Propositions de réponse } & Groupe « CO » & Groupe « AS » \\
\hline Pas de solutions et la situation s'est empirée & 0 & $15(10,1 \%)$ \\
\hline Pas de changement & $7(13,46 \%)$ & $22(14,86 \%)$ \\
\hline Légère amélioration & $12(23,08 \%)$ & $45(30,40 \%)$ \\
\hline Résolue comme vous l'avez désiré & $33(63,46 \%)$ & $65(43,92 \%)$ \\
\hline
\end{tabular}

\subsection{Evaluation des risques}

Les sujets ont évalué l'intensité de 13 risques, sur une échelle allant de 0 à 4 . De nombreuses différences statistiquement significatives sont présentes entre les deux groupes (cf tableau III). Les jeunes du groupe « AS » évaluent plus intensément les risques liés à leur propre bien-être et à leur avenir. Alors que les risques qui concernent les proches et la possibilité de les blesser sont plus marqués dans le groupe « $\mathrm{CO} »$. Lors de la divulgation de 
leur homosexualité, les jeunes homosexuels ne présentent donc pas les mêmes craintes qu'au cours des autres situations stressantes qu'ils peuvent rencontrer. Cependant, même si la nature des risques diffère, le stress perçu est similaire entre les deux groupes puisque le score total moyen est statistiquement identique entre les deux groupes.

Tableau III : Evaluation de l'intensité des risques encourus au cours de la situation stressante, en fonction des deux groupes de sujets (moyennes, écarts-types et $t$ de student).

\begin{tabular}{|l|c|c|c|c|}
\hline & Groupe « AS » & Groupe « CO » & $\mathbf{t}_{(198)}$ & $\mathbf{p}$ \\
\hline $\begin{array}{l}\text { a. Nuire au bien-être physique, et } \\
\text { à la santé d'un de vos proches }\end{array}$ & $1,24(1,62)$ & $0,81(1,27)$ & 1,76 & $\mathrm{NS}$ \\
\hline b. Faire de la peine à un proche & $2,32(1,70)$ & $3,10(1,32)$ & 2,96 & 0,003 \\
\hline $\begin{array}{l}\text { c. Nuire à votre propre santé } \\
\text { physique, à votre sécurité ou à } \\
\text { votre bien-être }\end{array}$ & $2,68(2,27)$ & $1,69(1,60)$ & 2,90 & 0,004 \\
\hline $\begin{array}{l}\text { d. Perturber les habitudes d'un } \\
\text { proche }\end{array}$ & $1,45(1,61)$ & $2,58(1,35)$ & 4,53 & 0,0001 \\
\hline $\begin{array}{l}\text { e. Ne pas réussir un but important } \\
\text { au travail }\end{array}$ & $1,32(1,67)$ & $0,17(0,59)$ & 4,87 & 0,0001 \\
\hline $\begin{array}{l}\text { f. De diminuer vos ressources } \\
\text { financières }\end{array}$ & $0,91(1,42)$ & $0,13(0,49)$ & 3,86 & 0,0001 \\
\hline $\begin{array}{l}\text { g. Perdre l'affection de quelqu'un } \\
\text { d'important pour vous }\end{array}$ & $1,97(1,81)$ & $3,44(1,02)$ & 5,55 & 0,0001 \\
\hline h. Perdre votre amour propre & $1,57(1,55)$ & $1,00(1,40)$ & 2,36 & 0,02 \\
\hline $\begin{array}{l}\text { i. } \text { Apparaître comme quelqu'un } \\
\text { d'insouciant }\end{array}$ & $1,03(1,48)$ & $0,85(1,27)$ & 0,78 & $\mathrm{NS}$ \\
\hline $\begin{array}{l}\text { j. Apparaître comme quelqu'un } \\
\text { sans moralité }\end{array}$ & $1,01(1,46)$ & $1,17(1,40)$ & 0,69 & $\mathrm{NS}$ \\
\hline $\begin{array}{l}\text { k. Perdre l'approbation ou le } \\
\text { respect de quelqu'un } \\
\text { d'important pour vous }\end{array}$ & $1,90(1,67)$ & $2,94(1,32)$ & 4,09 & 0,0001 \\
\hline $\begin{array}{l}\text { l. Perdre le respect pour } \\
\text { quelqu'un d'autre }\end{array}$ & $0,94(1,44)$ & $1,48(1,69)$ & 2,23 & 0,03 \\
\hline m. Vous montrer incompétent & $1,47(1,62)$ & $0,25(0,71)$ & 5,24 & 0,0001 \\
\hline $\begin{array}{l}\text { Score total des risques encourus } \\
\text { A, }\end{array}$ & $1,53(0,75)$ & $1,61(0,62)$ & 0,73 & $\mathrm{NS}$ \\
\hline
\end{tabular}




\subsection{Evaluation des possibilités d'action}

Les participants ont estimé les capacités d'action qu'ils jugeaient avoir sur la situation. Pour les deux groupes, la possibilité d'action qui est privilégiée est l'acceptation. Parmi les quatre possibilités d'action proposées, deux sont significativement différentes entre les deux groupes (cf tableau IV). En moyenne, les sujets du groupe «AS » jugent avoir plus de possibilités d'action sur la situation stressante que ceux du groupe « $\mathrm{CO} »$. Lorsque les jeunes homosexuels se confrontent à une annonce hautement stressante de leur homosexualité ils ont donc un contrôle perçu plus faible que lors des autres situations stressantes.

Tableau IV : Evaluation de l'intensité de chaque possibilités d'action face à la situation stressante, en fonction des deux groupes de sujets (moyennes, écarts-types et $t$ de student).

\begin{tabular}{|l|c|c|c|c|}
\hline & $\begin{array}{c}\text { Groupe « AS » } \\
\mathbf{N}=\mathbf{1 4 8}\end{array}$ & $\begin{array}{c}\text { Groupe « CO » } \\
\mathbf{N}=\mathbf{5 2}\end{array}$ & $\mathbf{t}_{(\mathbf{1 9 8})}$ & $\mathbf{p}$ \\
\hline $\begin{array}{l}\text { a. Vous pouviez la changer ou } \\
\text { y faire quelque chose }\end{array}$ & $1,51(1,48)$ & $0,88(1,15)$ & 2,76 & 0,006 \\
\hline b. Vous deviez l'accepter & $2,82(1,43)$ & $3,23(1,17)$ & 1,84 & $\mathrm{NS}$ \\
\hline $\begin{array}{l}\text { c. Vous deviez en savoir plus } \\
\text { avant d'agir }\end{array}$ & $1,72(1,56)$ & $0,94(1,27)$ & 3,25 & 0,001 \\
\hline $\begin{array}{l}\text { d. Vous deviez vous retenir de } \\
\text { faire ce que vous auriez } \\
\text { voulu. }\end{array}$ & $1,74(1,65)$ & $1,33(1,54)$ & 1,57 & $\mathrm{NS}$ \\
\hline \begin{tabular}{l} 
Total \\
\hline
\end{tabular} & $1,95(0,83)$ & $1,60(0,69)$ & 2,74 & 0,007 \\
\hline
\end{tabular}

\subsection{Risque suicidaire}

Au sein de l'ensemble de l'échantillon, 38,5\% (77 sujets) ont pensé à mettre fin à leurs jours suite à la situation la plus stressante de leur vie, 24\% (49 jeunes) ont déclaré avoir 
imaginé un scénario suicidaire et 15\% (30 jeunes) seraient passés à l'acte. Ces résultats mettent en évidence les liens forts chez les jeunes homosexuels entre la confrontation à une situation hautement stressante et leur risque suicidaire. Au cours de la divulgation de leur homosexualité (groupe « $\mathrm{CO} »$ ), le risque suicidaire est légèrement plus élevé que lors des autres situations stressantes (groupe «AS») mais la répartition des effectifs aux deux modalités de réponse n'est pas statistiquement différente entre les deux groupes (cf tableau V).

Tableau V : Comparaison des effectifs et de la fréquence des sujets aux items concernant les idées, scénarios et conduites suicidaires au moment de la situation stressante

\begin{tabular}{|c|c|c|c|c|c|}
\hline \multirow[b]{2}{*}{ Items } & \multicolumn{2}{|c|}{$\begin{array}{c}\text { Sujets ayant } \\
\text { répondu « oui » }\end{array}$} & \multicolumn{2}{|c|}{$\begin{array}{c}\text { Sujets ayant } \\
\text { répondu « non » }\end{array}$} & \\
\hline & $\begin{array}{l}\text { groupe } \\
« \mathrm{CO} »\end{array}$ & $\begin{array}{l}\text { groupe } \\
« A S »\end{array}$ & $\begin{array}{l}\text { groupe } \\
« \mathrm{CO} »\end{array}$ & $\begin{array}{l}\text { groupe } \\
\text { «AS» }\end{array}$ & \\
\hline $\begin{array}{c}\text { J'ai pensé à mettre fin à mes jours } \\
\text { durant un bref instant }\end{array}$ & $\begin{array}{c}24 \\
(46,2 \%)\end{array}$ & $\begin{array}{c}53 \\
(35,8 \%)\end{array}$ & $\begin{array}{c}28 \\
(53,8 \%)\end{array}$ & $\begin{array}{c}95 \\
(64,2 \%)\end{array}$ & $\begin{array}{c}\chi_{1}^{2}=1,74 \\
\mathrm{p}=\mathrm{NS}\end{array}$ \\
\hline $\begin{array}{l}\text { J'ai imaginé un scénario pour me } \\
\text { tuer et je voulais vraiment mourir }\end{array}$ & $\begin{array}{c}15 \\
(28,8 \%)\end{array}$ & $\begin{array}{c}34 \\
(23 \%)\end{array}$ & $\begin{array}{c}37 \\
(71,2 \%)\end{array}$ & $\begin{array}{c}114 \\
(77 \%)\end{array}$ & $\begin{array}{c}\chi_{1}^{2}=0,72 \\
\mathrm{p}=\mathrm{NS}\end{array}$ \\
\hline $\begin{array}{c}\text { J'ai tenté de me tuer et j'espérais } \\
\text { vraiment mourir }\end{array}$ & $\begin{array}{c}9 \\
(17,3 \%)\end{array}$ & $\begin{array}{c}21 \\
(14,2 \%)\end{array}$ & $\begin{array}{c}43 \\
(82,7 \%)\end{array}$ & $\begin{array}{c}127 \\
(85,8 \%)\end{array}$ & $\begin{array}{c}\chi_{1}^{2}=0,30 \\
\mathrm{p}=\mathrm{NS}\end{array}$ \\
\hline
\end{tabular}

Nous n'observons pas de différence statistiquement significative entre les deux groupes en ce qui concerne leurs symptômes anxieux et dépressifs ainsi que leur risque suicidaire (cf tableau VI). Selon les normes établies par les auteurs de la HADS, pour les sujets ayant des scores supérieurs à 7 à l'échelle d'anxiété et/ou de dépression, un état dépressif et/ou anxieux 
peut être soupçonné. Or, le score moyen à l'échelle d'anxiété pour nos deux groupes est supérieur à cette valeur de référence, un état anxieux peut donc être soupçonné chez une grande partie de nos participants, et ceci sans distinction de groupe. De plus, au sein de notre échantillon total, $61,5 \%$ des sujets ont un score à l'échelle d'anxiété supérieur ou égal à 8 . Par contre, le score moyen à l'échelle de dépression est beaucoup plus faible. Un état dépressif peut être suspecté (scores supérieurs à 7) chez seulement 19,5\% des sujets. En ce qui concerne la SBQ-R, les auteurs considèrent qu'au sein de la population générale, un score supérieur ou égal à 7 signifie que le sujet présente un risque suicidaire élevé. Or, pour cette variable également, le score moyen des participants de nos deux groupes est supérieur à cette norme. Parmi l'ensemble des jeunes homosexuels interrogés, 55,6\% ont un score supérieur ou égal à 7 et présentent donc un risque suicidaire élevé.

Tableau VI : Scores à la SBQ-R et aux échelles de dépression et d'anxiété de la HADS (moyennes, écarts-types et $t$ de student)

\begin{tabular}{|l|c|c|c|c|}
\hline & $\begin{array}{c}\text { Groupe « AS » } \\
\mathbf{N}=\mathbf{1 4 8}\end{array}$ & $\begin{array}{c}\text { Groupe « CO » } \\
\mathbf{N}=\mathbf{5 2}\end{array}$ & $\mathbf{t}_{\mathbf{( 1 9 8 )}}$ & $\mathbf{p}$ \\
\hline Score d'anxiété (HADS) & $9,44(4,60)$ & $8,60(3,85)$ & 1,18 & NS \\
\hline Score de dépression (HADS) & $4,72(3,437)$ & $3,85(2,645)$ & 1,66 & NS \\
\hline Risque suicidaire (SBQ-R) & $7,67(3,74)$ & $7,48(3,15)$ & 0,33 & NS \\
\hline
\end{tabular}

\subsection{Modèle prédictif des idées suicidaires}

Nos résultats démontrent que lorsque les jeunes homosexuels s'exposent à une situation hautement stressante, les idées suicidaires sont fréquentes. Dans notre échantillon total, $38,85 \%$ des sujets affirment «avoir pensé à mettre fin à leurs jours » suite à la situation la plus stressante de leur vie. Afin d'identifier les variables permettant de prédire la présence 
d'idées suicidaires, une analyse de régression «pas à pas » a été réalisée, intégrant les différentes dimensions de la transaction stressante prises en considération dans cette recherche (cf tableau VII). Trois risques sont significativement prédictifs de la présence d'idées suicidaires («Perdre le respect pour quelqu'un d'autre », «Perdre l'affection de quelqu'un d'important pour vous », « Nuire à votre propre santé physique, à votre sécurité ou à votre bien-être »), il en est de même de leur évaluation de l'issue de la situation. Une situation stressante ayant une issue jugée défavorable par le sujet est prédictive d'idéations suicidaires. Les variables incluses dans ce modèle de régression $(F=13,43 ; p=0,0001)$ permettent d'expliquer près de 20,3\% de la présence des idées suicidaires ( $\mathrm{R}$ deux ajusté $=0,203$ ).

Tableau VII : Paramètres du modèle de régression des idées suicidaires lors de la situation stressante (variable dépendante) intégrant trois risques et l'issue de la situation (variables indépendantes)

\begin{tabular}{|c|c|c|c|c|c|}
\hline \multirow{2}{*}{ Variables } & \multicolumn{2}{|c|}{$\begin{array}{c}\text { Coefficients non } \\
\text { standardisés }\end{array}$} & $\begin{array}{c}\text { Coefficients } \\
\text { standardisés }\end{array}$ & \multirow{2}{*}{$\mathbf{t}$} & p \\
\cline { 2 - 5 } & B & $\begin{array}{c}\text { Erreur } \\
\text { standard }\end{array}$ & Bêta & \\
\hline $\begin{array}{c}\text { Perdre le respect pour quelqu'un } \\
\text { d'autre }\end{array}$ & 0,08 & 0,02 & 0,25 & 3,77 & 0,0001 \\
\hline $\begin{array}{c}\text { Perdre l'affection de quelqu'un } \\
\text { d'important pour vous }\end{array}$ & 0,07 & 0,02 & 0,24 & 3,55 & 0,0001 \\
\hline $\begin{array}{c}\text { Nuire à votre propre santé physique, à } \\
\text { votre sécurité ou à votre bien-être }\end{array}$ & 0,04 & 0,01 & 0,16 & 2,44 & 0,02 \\
\hline Résultats de la situation & $-0,05$ & 0,02 & $-0,17$ & 2,58 & 0,01 \\
\hline
\end{tabular}

\section{Discussion}


Nos résultats montrent que quasiment tous les participants ont déjà annoncé au moins une fois leur homosexualité (seulement 5 jeunes ne l'ont jamais dévoilé à qui que ce soit). Mais cette révélation n'a pas généré le même stress chez tous. Pour $26 \%$ des jeunes interrogés, l'annonce de leur homosexualité a constitué la situation la plus stressante de leur vie (groupe « $\mathrm{CO} »)$ et pour la moitié d'entre eux, c'est la divulgation à leurs parents qui a été la plus stressante. Les sujets restants ont évoqué d'autres situations (groupe « AS »), mais ils ont presque tous déclaré avoir été stressés au moins une fois par la divulgation de leur homosexualité (seulement 10 sujets ne l'ont jamais été). La divulgation de son homosexualité est donc fréquemment source de stress, même si l'intensité du stress est variable selon les sujets et les personnes à qui l'annonce est adressée.

Lors d'une annonce hautement stressante de leur homosexualité (groupe « $\mathrm{CO} »)$, les jeunes homosexuels ont essentiellement peur de blesser leurs proches. A l'inverse, au cours des autres situations stressantes (groupe « AS »), leurs craintes sont centrées sur l'impact que la situation peut avoir sur leur propre bien-être. Ces divergences dans l'évaluation cognitive des risques encourus illustrent une des spécificités du stress durant cette divulgation. Au cours de cette annonce, le stress perçu est associé à l'entourage plus qu'à l'individu. En effet, dévoiler son homosexualité nécessite de s'exposer au regard de l'autre et à un éventuel rejet. L'inadéquation des homosexuels à la «norme» qu'est l'hétérosexualité les conduit à s'exposer à de possibles réactions négatives, et ceci dans différents domaines de leur vie, que ce soit au sein de leur famille (Willoughby, Malik, \& Lindahl, 2006b) ou de leur milieu scolaire (Chamberland, Gilbert, Julien, Otis, \& Ryan, 2010). Cette crainte du rejet constitue une des principales motivations des jeunes homosexuels à cacher leur homosexualité (D'Augelli, Grossman, Starks, \& Sinclair, 2010; Pachankis, 2007). De plus, lorsque les jeunes homosexuels s'exposent à une révélation très stressante de leur homosexualité, ils jugent avoir 
peu de moyen d'agir sur leur annonce. La faiblesse des moyens d'intervention peut influencer leur sentiment de contrôle et donc impacter sur leur stress. Or, cette divulgation fait l'objet d'une plus grande préparation et de plus d'initiative que les autres situations stressantes. Ces données apparaissent contradictoires avec le fait de penser avoir peu de moyens d'action. Nous pouvons penser que ces jeunes considèrent avoir peu de moyens d'agir sur la réaction de leurs proches plus que sur la situation en elle même.

A travers cette recherche, nous avons également montré que lorsque les jeunes homosexuels se confrontent à une situation hautement stressante, leur risque suicidaire est élevé, et ceci quelle que soit la situation. En effet, nous n'observons pas de différence statistiquement significative entre les deux groupes, alors même que nous nous attendions à observer un risque suicidaire plus marqué lors de la divulgation de leur homosexualité. Ces résultats illustrent les liens étroits existants entre le stress et le risque suicidaire chez les jeunes homosexuels, indépendamment de la situation stressante. Par conséquent, la divulgation de son homosexualité en soi ne constitue pas une situation durant laquelle le risque suicidaire est plus élevé qu'au cours des autres situations stressantes. A partir de ces résultats, nous pouvons penser que c'est l'accumulation des stresseurs qui constituerait un facteur de risque, et ceci indépendamment de la nature du stresseur (stresseurs généraux ou spécifiques). Cette hypothèse va dans le sens des données issues de la littérature qui précisent que les tentatives de suicide chez les jeunes adultes sont étroitement liées à la multiplication des événements stressantes (Hawton \& Harriss, 2008). Selon le modèle des «stresseurs des groupes minoritaires », cette accumulation serait plus marquée chez les jeunes homosexuels, les stresseurs spécifiques (l'annonce de leur homosexualité, les propos homophobes...), s'additionnant aux stresseurs rencontrés par la population générale. Cependant, l'absence d'un 
groupe contrôle composé de personnes hétérosexuelles ne nous permet pas d'appréhender en finesse les spécificités du stress en fonction de l'orientation sexuelle.

La présence d'idéations suicidaires est étroitement liée à la manière dont les jeunes homosexuels évaluent les risques qu'ils encourent lors de la situation stressante. Plus ils vont considérer que cette situation risque de nuire à leur propre bien-être, de modifier le respect et l'affection de leurs proches, plus ils auront de risque de présenter des idées suicidaires. Ces conclusions ouvrent un nouveau champ de travail dans la prévention du suicide chez les jeunes homosexuels. Elles mettent en avant l'importance d'intégrer des éléments associés à la gestion du stress dans la prévention du suicide au sein de cette population. De même, l'issue de la situation est également une dimension prédictive des idéations suicidaires. Les situations évoquées impliquent très majoritairement le cercle familial, ces résultats confirment que famille et le soutien des parents constituent d'importants facteurs de protection contre le suicide chez les jeunes homosexuels (Eisenberg \& Resnick, 2006; Kidd et al., 2006). Nos résultats sont plutôt encourageants, puisque majoritairement, les sujets ont évalué positivement l'issue de la situation, et ceci d'autant plus lors de la divulgation de leur homosexualité. Cependant, ces résultats doivent être nuancés par l'aspect rétrospectif de notre questionnaire. En effet, il s'est déroulé en moyenne 3 ans entre la passation du questionnaire et la situation en elle-même, ce qui a pu conduire certains sujets à évaluer une issue satisfaisante au moment de la passation, alors même qu'ils auraient jugé la finalité insatisfaisante dans le passé. Alors que la littérature met l'accent sur la fréquence des réactions négatives suite à l'annonce (Savin-Williams, 2003a; Willoughby \& Malik, 2006a), nos données laissent penser que les attitudes des proches évoluent positivement avec le temps, ce qui a été également démontré dans d'autres recherches (Charbonnier \& Graziani, 2011; D'Augelli, Grossman, Starks, \& Sinclair, 2010). Cette divulgation implique de la part des 
parents un ajustement progressif, qu'ils se dégagent de leurs stéréotypes concernant l'homosexualité mais aussi de leur sentiment de culpabilité (Floyd, Stein, Harter, Allison, \& Nye, 1999). Par conséquent, au vu de la fréquence des réactions négatives observée dans la littérature et de l'aspect prédictif d'une issue défavorable sur la présence d'idées suicidaires démontré dans notre recherche, le développement de campagnes de prévention et d'information, plus directement destinées aux parents (qui sont les personnes auprès de qui la divulgation apparait être la plus stressante) constitue un axe de prévention essentiel.

\section{- Limites de la recherche}

Cette recherche présente un certain nombre de limites qui imposent de nuancer certains résultats. Nos données montrent que lorsque les jeunes homosexuels se confrontent à une situation hautement stressante, leur risque suicidaire est élevé, ce qui illustre les liens étroits entre le stress et le risque suicidaire chez les jeunes homosexuels. Cependant, en l'absence d'un groupe de comparaison comprenant des personnes hétérosexuelles, nous ne pouvons pas affirmer avec certitude que cette vulnérabilité au stress n'est liée qu'à leur appartenance à une minorité sexuelle.

De plus, notre échantillon et notre recrutement peuvent également être critiqués. Le recours à internet n'induisant pas de contacts « réels » avec les participants, il peut laisser plus de place au «mensonge» qu'un questionnaire administré en face en face. Cependant, la véracité des réponses des sujets lors des recherches est une question qui se pose dès l'instant où un individu est interrogé et doit donner son avis, évaluer un fait. En ce qui concerne plus précisément le recours à internet, plusieurs auteurs (LaRose, Lin, \& Eastin, 2003; Mastro, Eastin, \& Tamborini, 2002) considèrent que certaines personnes choisissent de communiquer 
par internet pour lutter contre leur humeur dysphorique, notamment en ayant recours à des forums. Les sujets de cette recherche étant des personnes inscrites sur des sites de rencontres, il est donc possible qu'ils soient plus en souffrance que la majorité des homosexuels. Dans le même temps, Byrne et al. (2008) constatent que plus la communication établie par le biais d'e-mails et de forums de discussion est conséquente, plus les symptômes dépressifs diminuent. Il est donc difficile de déterminer si les personnes présentes sur ce site sont plus ou moins en souffrance que celles qui n'y sont pas.

Nous pouvons également nous interroger sur les motivations des sujets à participer à cette recherche. Les personnes qui sont le plus en souffrance ont pu être plus motivés à réaliser ce type de démarche, que ce soit dans le but de partager leur mal-être ou encore de rechercher une amélioration à leur état, ce qui conduirait à nuancer certains résultats, notamment les scores élevés à la HADS et la SBQ-R. Mais là encore, cette question peut se poser pour toute recherche impliquant une participation volontaire des sujets.

\section{Conclusion et pistes d'accompagnement}

Pour conclure, dans le cas d'un jeune venant consulter pour être aidé par rapport à la divulgation de son homosexualité, deux axes thérapeutiques doivent être privilégiés : la diminution du stress perçu (notamment en l'accompagnant dans la prise de recul concernant les risques qu'il juge encourir) et l'augmentation du contrôle perçu (en élaborant par exemple avec lui diverses stratégies de résolution de problème). De plus, les cognitions associées à cette annonce doivent être repérées, tout comme les croyances irrationnelles, les attentes et les objectifs du patient (Millêtre, 2004). Cette démarche apparait d'autant plus importante que la divulgation de leur homosexualité constitue une étape charnière dans leur construction 
identitaire et l'acceptation de leur orientation sexuelle (Rosario, Schrimshaw, \& Hunter, 2004). Des prises en charge s'inspirant des programmes de gestion du stress et reposant sur les techniques cognitives et comportementales (Légeron, 2002) nous apparaissent pertinentes pour les aider à affronter des situations très stressantes. Ce type de prise en charge pourrait également favoriser la prévention de leurs conduites suicidaires suite à l'exposition à une situation stressante. Ces conclusions ouvrent donc un nouveau champ de travail dans la prévention du suicide chez les jeunes homosexuels.

Les spécificités du stress présentes lors de la divulgation de leur homosexualité démontrent la nécessité de connaitre les difficultés propres aux homosexuels comme le précise le guide de recommandation pour la psychothérapie auprès des patients gays, lesbiennes et bisexuels (American Psychological Association, 2011). Or, il existe en France très peu de programme de formation abordant ces questions et les professionnels travaillant auprès de jeunes disposent souvent d'assez peu de connaissances concernant l'homosexualité et les stresseurs spécifiques liés à l'appartenance à une minorité sexuelle. De plus, même si la prise en charge peut être dans ses fondements sensiblement la même que celle qui peut être proposée aux personnes hétérosexuelles, elle implique que le thérapeute réfléchisse à ses propres croyances et préjugés envers l'homosexualité, l'accompagnement pouvant être gravement affecté par les attitudes négatives, implicites ou explicites, du professionnel. Il se doit de remédier à ses propres préjugés en les remplaçant par des connaissances scientifiques sur l'homosexualité (Fortier \& Julien, 2003). Cette réflexion est un préalable nécessaire pour créer un environnement clinique empathique et compréhensif (Anhalt, Morris, Scotti, \& Cohen, 2003). La question de l'orientation sexuelle étant rarement le motif de consultation évoqué, certains auteurs (Frappier, Girard, Meilleur, \& Ryan, 1997) préconisent d'aborder systématiquement avec les adolescents leur orientation sexuelle, à l'aide de questions claires 
et sans allusion, telles que "il arrive que des jeunes de ton âge aient des activités sexuelles avec une personne du même sexe, as-tu déjà eu de telles activités?".

Plus globalement, des campagnes d'informations et des groupes de discussion à destination des adolescents centrés sur la diversité sexuelle, les stresseurs spécifiques des groupes minoritaires et les lieux dans lesquels ils peuvent en parler, pourraient favoriser la réduction du sentiment d'isolement ressenti par certains jeunes et faciliter l'accès aux lieux de consultation pour ceux se retrouvant en souffrance mais ayant des craintes quant au fait de consulter. De plus, même si l'axe individuel a été privilégié dans cette recherche pour des raisons méthodologiques, l'annonce de son homosexualité est une divulgation adressée à une autre personne et l'entourage a donc un rôle essentiel dans cette situation particulière. Pour le plus grand nombre, c'est l'annonce faite à leur famille qui est la plus stressante. Par conséquent, une campagne d'information ayant pour cible les familles nous apparait tout aussi essentielle. De plus, l'issue de l'annonce étant prédictive des idées suicidaires, l'information aux familles pourrait donc également constituer un axe de prévention du suicide chez les jeunes homosexuels, les connaissances sur l'homosexualité favorisant l'ajustement et l'acceptation des parents (Ben-Ari, 1995).

\section{Références}

Adam, P. (2001). Dépression, tentatives de suicide et prise de risque parmi les lecteurs de la presse gay française. Paper presented at the Vulnérabilité des jeunes gays et lesbiennes et risque de suicide : Etat de la question et pistes de prévention. Synthèse de la journée d'étude organisée le 17 juillet 2001, Bruxelles.

American Psychological Association. (2011). Practice Guidelines for LGB Clients. Guidelines for Psychological Practice with Lesbian, Gay, and Bisexual Clients. Washington, DC: APA. 
Anhalt, K., Morris, T. L., Scotti, J. R., \& Cohen, S. H. (2003). Student perspectives on training in gay, lesbian, and bisexual issues: A survey of behavioral clinical psychology programs. Cognitive and Behavioral Practice, 10, 255-263.

Beck, F., Firdion, J. M., Legleye, S., \& Schiltz, M. A. (2010). Les minorités sexuelles face au risque suicidaire. Acquis des sciences sociales et perspectives. Saint Denis INPES.

Ben-Ari, A. (1995). The discovery that an offspring is gay: Parents', gay men's, and lesbians' perspectives. Journal of Homosexuality, 30, 89-112.

Bonet, L., Wells, B. E., \& Parsons, J. T. (2007). A positive look at a difficult time: A strength based examination of coming out for lesbian and bisexual women. Journal of LGBT Health Research, 3, 7-14.

Byrne, S., Morgan, S., Fitzpatrick, C., Boylan, C., Crowley, S., Gahan, H., et al. (2008). Deliberate self-harm in children and adolescents: a qualitative study exploring the needs of parents and carers. Clinical Child Psychology and Psychiatry, 13, 493-504.

Chamberland, L., Gilbert, E., Julien, D., Otis, J., \& Ryan, B. (2010). L'impact de l'homophobie et de la violence homophobe sur la persévérance et la réussite scolaires. Rapport de recherche, programme actions concertées : ministère de l'Éducation, du Loisir et du Sport (MELS) et Fonds Québécois de la recherche sur la société et la culture (FQRSC). Montréal: Université du Québec à Montréal.

Charbonnier, E., \& Graziani, P. (2011). La perception de jeunes lesbiennes et gais concernant l'attitude de leurs parents à l'égard de leur homosexualité. Revue canadienne de santé mentale communautaire, 30, 31-46.

Coyne, J. C., \& Lazarus, R. S. (1980). Cognitive style, stress perception and coping. In I. L. Kutasha \& L. B. Schlesinger (Eds.), Hand book of stress and anxiety : Knowledge, theory and treatment (pp. 144-158). San Francisco: Jossey Bass.

D'Augelli, A. R., Grossman, A. H., Starks, M. T., \& Sinclair, K. O. (2010). Factors associated with parents' knowledge of gay, lesbian, and bisexual youths' sexual orientation. Journal of GLBT family studies, 6(2), 178-198.

D'Augelli, A. R. (2002). Mental health problems among lesbian, gay, and bisexual youths ages 14 to 21. Clinical child psychology and psychiatry, 7(3), 433-456

Eisenberg, M. E., \& Resnick, M. D. (2006). Suicidality among gay, lesbian and bisexual youth: The role of protective factors. Journal of adolescent health, 39, 662-668.

Fenner, Y., Garland, S. M., Moore, E. E., Jayasinghe, Y., Fletcher, A., Tabrizi, S. N., et al. (2012). Web-Based recruiting for Health Research Using a Social Networking Site : An Exploratory Study. Journal of Medical Internet Research, 14(1), e20.

Floyd, F. J., \& Stein, T. S. (2002). Sexual orientation identity formation among gay, lesbian and bisexual youths: Multiple patterns of milestone experiences. Journal of Research on Adolescence, 12, 167-191.

Floyd, F. J., Stein, T. S., Harter, K. S. M., Allison, A., \& Nye, C. L. (1999). Gay, lesbian, and bisexual youths: separation-individuation, parental attitudes, identity consolidation, and well-being. Journal of youth and adolescence, 28, 719-739. 
Fortier, C., \& Julien, D. (2003). Les psychothérapies de conversion pour les personnes gaies, lesbiennes et bisexuelles : enjeux éthiques et déontologiques. Canadian Psychology 44(4), 332-350.

Frappier, J. Y., Girard, M., Meilleur, D., \& Ryan, B. (1997). Orientation sexuelle et homosexualité à l'adolescence. Approches, soins, prévention. In P. A. Michaud, P. Alvin, J. P. Deschamps, J. Y. Frappier, D. Marcil \& A. Turz (Eds.), La santé des adolescents : approche, soins, prévention. Montréal, Paris, Lausanne: Presses de l'Université de Montréal, Doin, Payot

Garofalo, R., \& Katz, E. (2001). Health care issues of gay and lesbian youth. Current Opinion in Pediatrics, 13, 298-302.

Graziani, P., Rusinek, S., Servant, D., Hautekèete-Sence, D., \& Hautekèete, M. (1998). Validation Française du Questionnaire de Coping "Ways of Coping Check-list Revised (W.C.C.-R.) et analyse des événements stressants du quotidien. Journal de Thérapie Cognitivo-Comportementale, 8(3), 1-13.

Hawton, K., \& Harriss, L. (2008). Deliberate self-harm by under-15-year-olds: characteristics, trends and outcome. The Journal of Child Psychology and Psychiatry, 49, 441-448.

Huebner, D. M., Rebchook, G. M., \& Kegeles, S. M. (2004). Experiences of harassment, discrimination, and physical violence among young gay andbisexual men. American Journal of Public Health, 94(7), 1200-1203.

Iwasaki, Y., \& Ristock, J. L. (2007). The nature of stress experienced by lesbians and gay men. Anxiety Stress Coping, 20(3), 299-319.

Jouvin, E., Beaulieu-Prévost, D., \& Julien, D. (2007). Minorités sexuelles : des populations plus exposées que les autres ? . In F. Beck, P. Guilbert \& A. Gautier (Eds.), Baromètre santé 2005 (pp. 355-367). Paris: INPES.

Julien, D. (2000). Famille d'origine et homosexualité. In M. Simard \& J. Alary (Eds.), Comprendre la famille : actes du 5e symposium québécois de recherche sur la famille (pp. 210-222). Québec: Presse Universitaire du Québec.

Kidd, S., Henrich, C. C., Brookmeyer, K. A., Davidson, L., King, R. A., \& Shahar, G. (2006). The social context of adolescent suicide attempts: Interactive effects of parent, peer, and school social relations. Suicide and Life-Threatening Behavior, 36(4), 386-395.

Kitts, R. L. (2005). Gay adolescents and suicide: Understanding the association. Adolescence, 40(159), 621-628.

LaRose, E., Lin, C., \& Eastin, M. S. (2003). Unregulated Internet usage : addiction, habit or deficient self-regulation? . Media Psychology, 5, 225-253.

Lazarus, R. S. (1966). Psychological stress and coping process. New York: McGraw-Hill.

Lazarus, R. S., \& Folkman, S. (1984). Stress, Appraisal and Coping. New York: Springer Publishing.

Légeron, P. (2002). La psychologie du stress et les stratégies de gestion du stress. Annales de Cardiologie et d'Angéiologie, 51, 95-102. 
Lhomond, B., \& Saurel-Cubizolles, M. J. (2003). Orientation sexuelle, violences contre les femmes et santé. Résultats de l'enquête nationale sur les violences envers les femmes en France. In C. Broqua, F. Lert \& Y. Souteyrand (Eds.), Homosexualités au temps du sida (pp. 107-130). Paris: ANRS.

Mastro, D., Eastin, M. S., \& Tamborini, R. (2002). Internet search behaviour and mood alteration: replication and expansion of selective exposure theory. Media Psychology, $4,157-172$.

Meyer, I. H. (2003). Prejudice, social stress, and mental health in lesbian, gay, and bisexual populations: conceptual issues and research evidence. Psychological Bulletin, 129(5), 674-697.

Meyer, I. H., Dietrich, J., \& Schwartz, S. (2008). Lifetime prevalence of mental disorders and suicide attempts in diverse lesbian, gay, and bisexual populations. American Journal of Public Health, 98(6), 1004-1006.

Meyer, I. H., \& Northridge, M. E. (2007). The Health of Sexual Minorities: Public Health Perspectives of Lesbian, Gay, Bisexual and Transgender Populations. New York: Springer.

Millêtre, B. (2004). Application des tcc au processus de " coming-out » chez une patiente homosexuelle. Journal de thérapie comportementale et cognitive, 14(3), 141-145.

Osman, A., Bagge, C. L., Gutierrez, P. M., Konick, L. C., Kopper, B. A., \& Barrios, F. X. (2001). The Suicidal Behaviors Questionnaire-Revised (SBQ-R): validation with clinical and nonclinical samples. Assessment, 8(4), 443-454.

Pachankis, J. E. (2007). The psychological implications of concealing a stigma: A cognitiveaffective-behavioral model. Psychological Bulletin, 133, 328-345.

Pouchieu, C., Castetbon, K., Galan, P., Hercberg, S., \& Touvier, M. (2012). Prise de compléments alimentaires évaluée par autoquestionnaire sur Internet ; étude NutriNetSanté. Revue d'Epidémiologie et de Santé Publique, 60, 95.

Rosario, M., Schrimshaw, E. W., \& Hunter, J. (2004). Gay-related stress and emotional distress among gay, lesbian, and bisexual youths: A longitudinal examination. Journal of Consulting and Clinical Psychology, 70, 967-975.

Saewyc, E. M. (2011). Research on Adolescent Sexual Orientation: Development, Health Disparities, Stigma, and Resilience. Journal of Research on Adolescence, 21, 256272.

Saewyc, E. M., Homma, Y., Skay, C. L., Bearinger, L. H., Resnick, M. D., \& Reis, E. (2009). Protective factors in the lives of bisexual adolescents in North America. American journal of public health, 99, 110-117.

Saewyc, E. M., Skay, C. L., Hynds, P., Pettingell, S., Bearinger, L. H., Resnick, M. D., et al. (2007). Suicidal ideation and attempts in North American school-based surveys: are bisexual youth at increasing risk? Journal of LGBT Health Research, 3(2), 25-36.

Savin-Williams, R. C. (2001). Mom, Dad, I'm gay: How families negotiate coming out. Washington, DC: American Psychological Association. 
Savin-Williams, R. C. (2003a). Lesbian, gay, and bisexual youths' relationships with their parents. In D. Kimmel \& L. Garnets (Eds.), Psychological perspectives on lesbian, gay, and bisexual experiences (pp. 299-326). New York: Columbia University Press.

Savin-Williams, R. C., \& Esterberg, K. G. (2000). Lesbian, gay, and bisexual families. In D. H. Demo, K. R. Allen \& M. A. Fine (Eds.), Handbook of family diversity (pp. 197215). New York: Oxford University Press.

Savin-Williams, R. C., \& Ream, G. L. (2003b). Sex variations in the disclosure to parents of same-sex attractions. Journal of Family Psychology, 17(3), 429-438.

Silenzio, V. M., Pena, J. B., Duberstein, P. R., Cerel, J., \& Knox, K. L. (2007). Sexual orientation and risk factors for suicidal ideation and suicide attempts among adolescents and young adults. American Journal of Public Health, 97(11), 2017-2019.

Velter, A. (2007). Rapport Enquête Presse Gay 2004. Saint-Maurice ANRS, INVS.

Willoughby, B. L. B., \& Malik, N. M. (2006a). Mothers, fathers, and their gay sons: A study of coming out. Paper presented at the annual meeting of the Society for the Scientific Study of Sexuality, Las Vegas, NV.

Willoughby, B. L. B., Malik, N. M., \& Lindahl, K. M. (2006b). Parental Reactions to Their Sons' Sexual Orientation Disclosures: The Roles of Family Cohesion, Adaptability, and Parenting Style. Psychology of men and masculinity, 7(1), 14-26.

Zigmond, A. S., \& Snaith, R. P. (1983). The hospital anxiety and depression scale. Acta Psychiatrica Scandinavica, 67(6), 361-370. 